\title{
FOLIATIONS IN SUPERGRAVITY
}

\author{
WAI KIN CHAN ${ }^{1}$, REYNALDO CASTILLO ${ }^{1}$ and KING FAI LAI ${ }^{2}$
}

(Received 27 February 1995)

\begin{abstract}
Following upon a previous paper [1] on the existence of chiral transformations in a foliated version of the Cremmer, Julia and Scherk model, we deduce a couple of interesting properties of the model. These are:

(i) $\mathrm{TM}_{4}$ is isomorphic to a quotient Lie pseudoalgebra on the algebra of basic functions in $M_{11}$;

(ii) There is a locally trivial fibration which exhibits $M_{11}$ as $M_{7} \times \mathscr{U}, \mathscr{U} \subset W$ and $W$ is the basic manifold of the foliation [5];

(iii) The chiral group of the model is identified as $C l^{x}\left(L, g_{L}\right) \times C l^{x}\left(Q, g_{Q}\right)$, the factors are respectively the multiplication groups of units in the Clifford algebras $C l^{x}\left(L, g_{L}\right)$ and $C l^{x}\left(Q, g_{Q}\right)$ and matching of this group with phenomenology is briefly discussed.
\end{abstract}

\section{Introduction}

It is generally accepted that one of the main problems with the Cremmer, Julia and Scherk Model (CJS) [2] is its inability to support chiral transformations [8]. In a previous paper [1], we show that this point can be remedied in such a way that none of the essential features of the 11-dimensional Kaluza-Klein mechanism in the original model needs to be changed. The starting point of the analysis is the observation that the metric tensor used in the original work for $M_{11}$ is bundle-line [6] and therefore suggests that $M_{11}$ has a foliation structure of dimension 7 and codimension 4 . The nature of the foliation is, of course, open. As a first step, we suggest that this foliation is a simple foliation in the sense of Molino [5] and is brought about by a global submersion of $M_{11}$ into $M_{4}$ with leaves difformorphic to $M_{7}$,

$$
f: M_{11} \rightarrow M_{4} \text {. }
$$

\footnotetext{
${ }^{1}$ Department of Computing and Information Systems, University of Western Sydney, Campbelltown, New South Wales 2560, Australia.

${ }^{2}$ School of Mathematics, University of Sydney, New South Wales 2000, Australia.

(C) Australian Mathematical Society 1999, Serial-fee code 0334-2700/99
} 
The restriction of $f$ to a leaf gives rise to a map $\varphi: M_{7} \rightarrow M_{4}$ which serves to relate happenings in the internal manifold to the physical world. It is important to note that the existence of this map is, by and large, implied by the bundle-like nature of the metric for $M_{11}$. In the second section, we show that the submersion (1) leads to some consequences on the tangent space of $M_{4}$. In the third section, we show that there is a locally trivial fibration $\pi_{b}: M_{11} \rightarrow W$, where $W$ is the basic manifold of the foliation. The fiber of this fibration is exactly $M_{7}$ and hence there is a foliation induced on it by (1). This foliation is a Lie foliation and its relation to the one assumed on $M_{7}$ in order to bring about chiral transformation is intriguing. At the present, we have not been able to resolve this problem. In the third section, we follow up on the analysis of [1] and identify the internal chiral group as $C l^{x}\left(L, g_{L}\right) \times C l^{x}\left(Q, g_{Q}\right)$, where the factors may be identified as automorphism groups of $C l^{x}\left(L, g_{L}\right)$ and $C l^{x}\left(Q, g_{Q}\right)$, where $L$ is the involutive subbundle of $T M_{7}$ which brings about the foliation in $M_{7}$, and $Q$ is the normal bundle. In the fourth section we conclude our analysis.

\section{Structural properties of the CJS model}

In the 11-dimensional Kaluza-Klein theory [8], one assumes that the ground state is not an 11-dimensional Minkowski space but a product space $M_{7} \times M_{4}$, where $M_{7}$ is a compact manifold of dimension 7 and $M_{4}$ is the physical world. Symmetries on $M_{7}$ will be observed as gauge symmetries in the 4-dimensional world $M_{4}$. If $\left(x^{m} ; m=l, \ldots, 7\right)$ are the coordinates of $M_{7}$ and $\left(T^{a} ; a=l, \ldots, N\right)$ are the generators of the symmetry group $G$ of $M_{7}$, then the action of $T^{a}$ on $x^{m}$ may be represented by $x^{m} \rightarrow x^{m}+K^{a m}(x)$, where $K^{a m}(x)$ is the Killing vector associated with the symmetry $T^{a}$. The massless excitation of $M_{7} \times M_{4}$ corresponds to the following ansatz for the metric tensor for $M_{11}$ (known normally as the DeWitt metric):

$$
\begin{aligned}
g_{A B} d z^{A} d z^{B}= & g_{u v}(y) d y^{u} d y^{v} \\
& +g_{m n}(x, y)\left(d x^{m}-K^{a m}(x) B_{u}^{a}(y) d y^{u}\right)\left(d x^{n}-K^{b n} B_{v}^{b} d y^{v}\right) .
\end{aligned}
$$

Here $A, B=1, \ldots, 11, u, v=1, \ldots, 4, a, b=1, \ldots, N ; m, n=1, \ldots, 7$. The metric tensor of the 4-dimensional world $M_{4}$ is denoted by $g_{u v}$ and $B_{u}^{a}(y)$ is the massless gauge field of the group $G$. This metric is bundle-like [6] and therefore suggests that $M_{11}$ carries a foliation of dimension 7 and codimension 4 . The inherent nature of this foliation is, at this point, open to model builders. As a first step, we suggest that it is a simple foliation [5] in the sense that the involutive subbundle that defines the foliation is the vertical tangent bundle of some surjective submersion

$$
f: M_{11} \rightarrow M_{4} .
$$


As in [1], this simple foliation is required to have leaves diffeomorphic to $M_{7}$. This gives rise to a map $\varphi=\left.f\right|_{M_{7}}$ mapping $M_{7}$ to $M_{4}$.

Now we are ready to state the first consequence of this simple foliation $F$ [4]. Let $\Delta$ be the involutive subbundle that defines the foliation $F$. Call a function $u \in C\left(M_{11}\right)$ basic if $X(u)=0$ for all $X \in \Gamma \Delta$ and denote the algebra of basic functions by $C\left(M_{11}\right)^{\Delta}$. Let

$$
L\left(M_{11}, \Delta\right)=\left\{X \in \Gamma T M_{11} \mid Y \in \Gamma \Delta \Rightarrow[X, Y] \in \Gamma \Delta\right\}
$$

be the normalizer [7] of $\Gamma \Delta$. in $\Gamma T M_{11}$. Elements of $L\left(M_{11}, \Delta\right)$ are called foliate vector fields [5]. Since $F$ is simple, the basic functions are precisely the pull-backs of functions on $M_{4}$ and the foliate vector fields are precisely those which project to vector fields in $M_{4}$. In terms of local coordinates this means the following. Let the metric in $M_{11}$ be expressed in the form:

$$
d s^{2}=\sum_{i, j=1}^{7} g_{i j}(x, f) \omega^{i} \omega^{j}+\sum_{\alpha, \beta=1}^{4} g_{\alpha \beta}(f) d f^{\alpha} d f^{\beta},
$$

where the 1-forms $\omega^{i}$ are of the form

$$
\omega^{i}=d x^{i}=\sum_{\alpha=1}^{4} a_{\alpha}^{i} d f^{\alpha}
$$

and $\left(\omega^{1}, \ldots, \omega^{7}, d f^{1}, \ldots, d f^{4}\right)$ is a basis of the cotangent space $T^{*} M_{11}$. The tangent space $T M_{11}$ is spanned by $\left(\partial / \partial x^{1}, \ldots, \partial / \partial x^{7}, v_{1}, \ldots, v_{4}\right)$ with

$$
v_{\alpha}=\frac{\partial}{\partial f^{\alpha}}-\sum_{i=1}^{4} a_{\alpha}^{i} \frac{\partial}{\partial x^{i}}
$$

Thus a basic function $u \in C\left(M_{11}\right)$ is one that does not depend on $x^{1}, \ldots, x^{7}$ and is of the form $u^{\sim} \circ f$ where $u^{\sim} \in C\left(M_{4}\right)$. A foliate vector field is one whose last four components do not depend on $x^{1}, \ldots, x^{7}$ and under $f$, such a vector field is projected into a vector field in $M_{\mathbf{4}}$.

$L\left(M_{11}, \Delta\right)$ is a Lie pseudoalgebra over $C\left(M_{11}\right)^{\Delta}$ and $\Gamma \Delta$ is an ideal. Let $l\left(M_{11}, \Delta\right)$ $=L(M, \Delta) / \Gamma \Delta$ be the quotient Lie pseudoalgebra, then $l\left(M_{11}, \Delta\right)$ is canonically isomorphic to $T M_{4}$ :

$$
l\left(M_{11}, \Delta\right) \cong T M_{4}
$$

This result depicts the tangent space of the physical world as a quotient Lie pseudoalgebra over the algebra of basic functions in $M_{11}$. In our opinion, it is interesting and may have consequences which may further prove or disprove the model. 
The next structural property of the foliated model may be explained as follows. If we assume that the foliate vector fields on $M_{11}$ span $T M_{11}$, then $F$ is known as a transversally complete foliation [5]. As explained in [5], $\forall$ neighbourhood $\theta$ of $x \in M_{11}$, one can find basic functions $f_{1}, \ldots, f_{q_{b}}$ whose differentials are linearly independent (in the neighbourhood under consideration). Consequently the system of equations

$$
d f_{1}=0, \quad \ldots, \quad d f_{q_{b}}=0
$$

is completely integrable. This system defines a foliation $F_{b}$ which is called a basic foliation of $\left(M_{11}, F\right)$. It has codimension $q_{b}$ and its leaves are unions of the leaves of $F$. The leaf space $M_{11} / F$ is Hausdorff and has the structure of a $q_{b}$-dimensional manifold, called $W$, the basic manifold of the foliation $F$. When $F$ is transversally complete, the closures of the leaves (these are just copies of $M_{7}$, since $M_{7}$ is closed) are fibers of a locally trivial fibration $\pi_{b}: M_{11} \rightarrow W$ onto the basic manifold $W$. On each fiber of $\pi_{b}$, the foliation induced by $F$ is a Lie foliation.

This result says that if $F$ is transversally complete then there are $q_{b}$ functions in $C\left(M_{4}\right)$ which, when pulled back to $M_{11}$, define level surfaces of $F_{b}$. Along directions transverse to these level surfaces (which are defined by physical objects) $M_{11}$ is locally trivial, that is, is of the form $M_{4} \times \mathscr{U}$ for some $\mathscr{U} \subset W$. This comes close to explaining mathematically what we mean by saying that in the ground state $M_{11}$ is of the form $M_{7} \times M_{4}$. It would be interesting to see what happens if we take the fibration $\pi$ instead of the product as the starting structure of $M_{11}$.

\section{The chiral group $C l_{p}^{x} \times C l_{q}^{x}$}

In [1], we show that if the $G$-action of Kaluza-Klein has the property that for every point $p \in M_{7}$ the tangent space to the orbit of the $G$-action has fixed dimension, then the orbits themselves define a foliation on $M_{7}$. The tangent space and the Riemannian metric of $M_{7}$ acquire the following orthogonal splitting;

$$
\begin{aligned}
T M_{7} & =L \oplus Q, \\
g_{M_{7}} & =g_{L} \oplus g_{Q} .
\end{aligned}
$$

The 7-dimensional Clifford algebra $\mathrm{Cl}\left(\mathrm{TM}_{7}, \mathrm{~g}_{\mathrm{M}_{7}}\right)$ is then isomorphic to the $Z_{2^{-}}$ graded tensor product $C l\left(L, g_{L}\right) \otimes^{\wedge} C l\left(Q, g_{Q}\right)$ [3]. It has been shown in [1] that if $M_{7}$ is spin then both $L$ and $Q$ are spin. It is then meaningful to talk about the spinor bundles $S(Q)$ and $S(L)$. Along with the orthogonal splittings (10) and (11), the spinor bundle on $M_{7}$, becomes a $Z_{2}$-graded tensor product $S(L) \otimes^{\wedge} S(Q)$ and spinors with definite helicities are of the form

$$
\psi_{\mathrm{int}}=\left(1 \pm \Omega_{p}\right) \psi \otimes\left(1 \pm \Omega_{q}\right) \sigma
$$


where $p$ and $q$ are respectively the dimension and the codimension of the foliation and $\Omega_{p}$ and $\Omega_{q}$ are the volume elements in the respective Clifford algebras and $\psi \in S(L)$ and $\sigma \in S(Q)$. Chiral transformations are generated by matrices of the form

$$
\Gamma_{i j}=2 \gamma_{i} \otimes \lambda_{j}
$$

Here $\left\{\gamma_{1}, \ldots, \gamma_{p}\right\}$ forms a basis for $C l\left(L, g_{L}\right)$ and $\left\{\lambda_{1}, \ldots, \lambda_{q}\right\}$ forms a basis for $C l\left(Q, g_{Q}\right)$. This set of matrices clearly generates a Lie group which is of the form $C l^{x}\left(L, g_{L}\right) \times C l^{x}\left(Q, g_{Q}\right)$. Here the group $C l^{x}(V, q)$ is the multiplicative group of units [3] in the Clifford algebra of the vector space $V$ and bilinear form $q$ and is defined to be the subset

$$
C l^{x}(V, q) \equiv\left\{\varphi \in C L(V, q) \mid \exists \varphi^{-1} \text { with } \varphi^{-1} \varphi=\varphi \varphi^{-1}=1\right\} .
$$

When the vector space is of finite dimension, say $n$, it is a Lie group of dimension $2^{n}$. In general there is an associated Lie algebra $c l^{x}(V, q)=C l(V, q)$ with Lie bracket given by

$$
[x, y]=x y-y x \text {. }
$$

The group of units acts naturally as automorphisms of the algebra, that is, there is a homomorphism

$$
\text { Ad }: C l^{x}(V, q) \rightarrow \operatorname{Aut}(C l(V, q))
$$

called the adjoint representation given by

$$
\operatorname{Ad}_{\phi}(x)=\phi x \phi^{-1}
$$

There is also a natural exponential mapping given by $\exp : c l^{x}(V, q) \rightarrow C l^{x}(V, q)$ defined by setting

$$
\exp (v)=\sum_{m=0}^{\infty} y^{m} / m !
$$

Thus we conclude that the chiral group of the model is $\operatorname{Cl}\left(L, g_{L}\right) \times \operatorname{Cl}\left(Q, g_{Q}\right)$. The dimension of this group is $2^{p}+2^{q}$ with $p+q=7$.

Just on the basis of simple minded dimension counting and assuming that $S U(3) \times$ $S U(2) \times U(1)$ is all the symmetry that we have got, this is clearly too large a number. Maybe there is a case to reduce the dimension of the internal manifold to, say, $p=3$, $q=3$ because then an internal symmetry group $U(3) \times U(2) \times U(1)$ would fit just nicely. 


\section{Conclusion}

In this paper, we have pointed out that the DeWitt metric for $M_{11}$ suggests that the 11-dimensional manifold in the CJS model is foliated. If one assumes that this foliation is simple in the sense of Molino, one arrives at interesting structural properties on top of the existence of chiral transformations. It would be extremely interesting to study these properties further in detail and to correlate them with observed phenomenology.

\section{References}

[1] W. K. Chan, K. F. Lai and R. Castillo, "Riemannian foliation in $N=1, D=11$ supergravity", $l l$ Nuovo Cimento 108B (1993) 739-754.

[2] E. Cremmer and and T. Scherk B. Julia, "Supergravity theory in 11 dimensions", Phys. Lett. B 76 (1978) 409-412.

[3] H. Lawson Jr. and M. Michelson, Spin geometry (Princeton University Press, 1989).

[4] K. Mackenzie, Lie groupoids and Lie algebras in differential geometry (Cambridge University Press, 1987)

[5] P. Molino, Riemannian Foliations, Progress in Math. 73 (Birkhauser, 1988).

[6] B. Reinhart, "Foliated manifolds with bundle-like metrics", Ann. Math. 69 (1959) 119-132.

[7] A. A. Sagle and R. E. Walde, Introduction to Lie groups and Lie algebras (Academic Press, 1973).

[8] E. Witten, "Search for a realistic Kaluza-Klein theory", Nucl. Phys. B 186 (1981) 412-428. 